\title{
Editorial: Plastics in the Environment: Understanding Impacts and Identifying Solutions
}

\author{
Montserrat Filella ${ }^{1 *}$, Andrew Turner ${ }^{2}$ and Hans Peter H. Arp ${ }^{3,4}$ \\ ${ }^{1}$ Department F.-A. Forel, University of Geneva, Geneva, Switzerland, ${ }^{2}$ School of Geography, Earth and Environmental Sciences, \\ University of Plymouth, Plymouth, United Kingdom, ${ }^{3}$ Norwegian Geotechnical Institute, Oslo, Norway, ${ }^{4}$ Norwegian University of \\ Science and Technology, Trondheim, Norway
}

Keywords: plastics, environment, impacts, pollution, effects

Editorial on the Research Topic

Plastics in the Environment: Understanding Impacts and Identifying Solutions

Plastics are one of the most widely used materials in the world which society will always be dependent on. This dependency has been clearly highlighted by the requirements for hygiene and protection during the recent global COVID pandemic (Adyel, 2020; Prata et al., 2020). Plastics are broadly integrated into today's lifestyle and are present in almost all consumer and industrial sectors and their production continues to increase (Geyer et al., 2017). Unfortunately, one of the characteristics of plastics that make them so useful-their durability-also ensures that they persist in the environment for very long periods of time. Additionally, and because of their low cost, many plastic objects have long been perceived as disposable. The consequence of this, coupled with the difficulty in developing effective waste management strategies, has been the ubiquitous contamination of the entire planet by plastic debris.

Even if proposed global actions to recycle more plastic or prevent the export of plastic waste to countries with poorly developed waste infrastructure through the Basel Convention are implemented, plastic emissions are expected to increase for the foreseeable future unless significant breakthroughs in plastic design or waste management are realized (Lau et al., 2020). Increasing emissions also imply that exposure to plastic pollution and its degradation products, like microplastics, nanoplastics, plastic additives, and other chemical leachates, will continue to increase. Such an accumulating plastic cocktail can result in complex and unpredictable impacts, including those on ecological processes (Rillig et al., 2021) or the global carbon cycle (Zhu, 2021).

Although the problem of plastic pollution was recognised several decades ago, research on plastics lost to the environment and their environmental and health impacts is now an extremely dynamic field involving a great deal of funding, support and effort. As an attempt to find solutions, there have been calls to integrate and introduce more biodegradable or recyclable plastics into the market in order to shift towards more sustainable supply chains. There is also some debate about which solutions make the most sense practically and economically, although it is likely that a combination of approaches may be required. An additional concern centres around the additives used in plastics, many of which are endocrine disrupting substances or otherwise harmful to the environment if released from the material. Chemical regulators, and particularly those in Europe, have become increasing active to make plastics safer and more recyclable, but clearly plastics are complex and diverse materials and understanding their complete life cycle and the (eco)toxicological implications of their extensive use and management is a highly justified, albeit difficult, task. 
A global, transdisciplinary problem requires a global, transdisciplinary response. Accordingly, the contributions in this Research Topic in Frontiers in Environmental and Marine Science: "Plastics in the Environment: Understanding Impacts and Identifying Solutions" cover a wide variety of aspects of plastic research and embrace a diversity of environment types, climates and habitats over a broad geographical extent (Europe, Asia, Africa, and the Arctic).

The importance of efficient but robust and comparable monitoring and detection methods is a key component of the Research Topic, and areas covered in this respect include the potential for remote sensing of litter using drones (EscobarSánchez et al.), the requirement for representative subsampling (Brandt et al.) and a critical review of techniques used to isolate microplastics from geogenic and biogenic material by chemical digestion (Pfeiffer and Fischer).

In many contributions, both contemporary and historical plastics are used to study environmental sources and distributions or to model transport and fate. For example, the general leakage of plastic to the environment and its subsequent distribution is explored using international, empirical data and its relation to drivers such as population density and land use (Schuyler et al.), while records of microplastics in marine sediments, ice cores and peat archives are used to attempt to unravel historical uses of plastics (Bancone et al.). Historical, aerial records of coastlines have also been combined with assessments of soil profiles, vegetation characteristics and type, and abundance of litter in a novel study examining the roles that plastics play in shaping coastal landscapes and habitats (Bastesen et al.). On beaches, emphasis is placed on the implications of quantifying plastic particles on a mass vs. number basis and considering both surface and buried debris (Ryan et al.),

\section{REFERENCES}

Adyel, T. M. (2020). Accumulation of Plastic Waste During COVID-19. Science 369, 1314-1315. doi:10.1126/science.abd9925

Geyer, R., Jambeck, J. R., and Law, K. L. (2017). Production, Use, and Fate of All Plastics Ever Made. Sci. Adv. 3, e1700782. doi:10.1126/sciadv.1700782

Lau, W. W. Y., Shiran, Y., Bailey, R. M., Cook, E., Stuchtey, M. R., Koskella, J., et al. (2020). Evaluating Scenarios toward Zero Plastic Pollution. Science 369, 1455-1461. doi:10.1126/science.aba9475

Prata, J. C., Silva, A. L. P., Walker, T. R., Duarte, A. C., and Rocha-Santos, T. (2020). COVID-19 Pandemic Repercussions on the Use and Management of Plastics. Environ. Sci. Technol. 54, 7760-7765. doi:10.1021/acs.est.0c02178

Rillig, M. C., Kim, S. W., Kim, T.-Y., and Waldman, W. R. (2021). The Global Plastic Toxicity Debt. Environ. Sci. Technol. 55 (5), 2717-2719. doi:10.1021/acs.est.0c07781 while in surface and subsurface seawater inputs of microplastics are calculated from urban runoff and waste water treatment plants (Schernewski et al.).

The weathering of plastics in the environment is considered in contributions that examine the fragmentation of expanded polystyrene into nano-sized particles by thermo-oxidation and hydrodynamic turbulence (Mattsson et al.) and that characterise microbial communities on conventional and biodegradable plastics in alpine and polar soils (Rüthi et al.). The impacts of both the fragmentation and aggregation of microplastics are also studied in the context of the feeding behaviour of freshwater zooplankton (Drago et al.). Further up the food chain, microplastics are profiled in long-lived marine animals (Meaza et al.) and are measured in a variety of animals, including commercially important pelagic fish (Bakir et al.; Haave et al.), with all studies linking observations to potential impacts on human exposure and health.

This eclectic collection of articles illustrates the wide diversity of environmental problems that arise from the mismanagement of plastics, and the many difficulties and challenges that scientists, managers, regulators, and stakeholders face in reducing or solving these problems. The transdisciplinarity of the subjects should also serve to inform scientists of the many and varied techniques and approaches that are currently available to guide future research towards understanding the diverse impacts of-and identifying the complex solutions to-plastic pollution.

\section{AUTHOR CONTRIBUTIONS}

All authors listed have made a substantial, direct, and intellectual contribution to the work and approved it for publication.

Zhu, X. (2021). The Plastic Cycle - an Unknown Branch of the Carbon Cycle. Front. Mar. Sci. 7, 609243. doi:10.3389/fmars.2020.609243

Conflict of Interest: The authors declare that the research was conducted in the absence of any commercial or financial relationships that could be construed as a potential conflict of interest.

Copyright (C) 2021 Filella, Turner and Arp. This is an open-access article distributed under the terms of the Creative Commons Attribution License (CC BY). The use, distribution or reproduction in other forums is permitted, provided the original author(s) and the copyright owner(s) are credited and that the original publication in this journal is cited, in accordance with accepted academic practice. No use, distribution or reproduction is permitted which does not comply with these terms. 